\title{
Search for Radions at LEP2
}

\section{Goetz Gaycken*†}

LLR-Ecole Polytechnique, France

E-mail: Goetz.gaycken@poly.in2p3.fr

\begin{abstract}
A new scalar resonance, called the radion, with couplings to fermions and bosons similar to those of the Higgs boson, is predicted in the framework of Randall-Sundrum models, proposed solutions to the hierarchy problem with one extra dimension. An important distinction between the radion and the Higgs boson is that the radion would couple directly to gluon pairs, and in particular its decay products would include a significant fraction of gluon jets. The radion has the same quantum numbers as the Standard Model (SM) Higgs boson, and therefore they can mix, with the resulting mass eigenstates having properties different from those of the SM Higgs boson. Existing searches for the Higgs bosons are sensitive to the possible production and decay of radions and Higgs bosons in these models. For the first time, searches for the SM Higgs boson and flavour-independent and decay-mode independent searches for a neutral Higgs boson are used in combination to explore the parameter space of the Randall-Sundrum model. In the dataset recorded by the OPAL experiment at LEP, no evidence for radion or Higgs particle production was observed in any of those searches at centre-of-mass energies up to $209 \mathrm{GeV}$. The results are used to set limits on the radion and Higgs boson masses. For all parameters of the RandallSundrum model, the data exclude masses below $58 \mathrm{GeV}$ for the mass eigenstate which becomes the Higgs boson in the no-mixing limit.
\end{abstract}

International Europhysics Conference on High Energy Physics

July 21st - 27th 2005

Lisboa, Portugal

* Speaker.

${ }^{\dagger}$ A footnote may follow. 

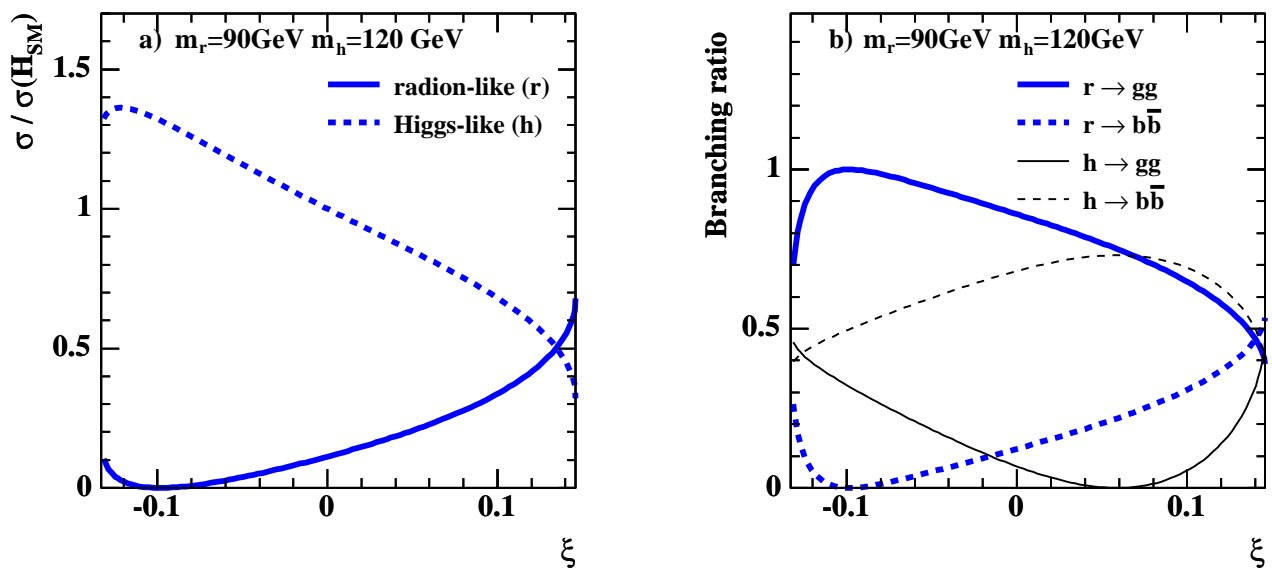

Figure 1: a) The cross-sections for the processes $\mathrm{e}^{+} \mathrm{e}^{-} \rightarrow \mathrm{Zr}$ or $\mathrm{Zh}$ of the radion-like and the Higgs-like state, $\mathrm{r}$ and $\mathrm{h}$, relative to the corresponding cross-section for a SM Higgs boson. c) The branching ratios of $r$ and $h$ into gluon pairs and $b \bar{b}$. The parameter $\Lambda_{\mathrm{W}}$ was chosen to be $300 \mathrm{GeV}$. The cross-sections and branching ratios of the Higgs-like state $\mathrm{h}$ are identical to those of a SM Higgs boson for $\xi=0$.

\section{Introduction}

The radion is a novel particle which appears in the Randall-Sundrum (RS) model [1]. This model was proposed to solve the hierarchy problem between the electroweak $\mathscr{O}(\mathrm{TeV})$ and the Planck mass scale $\mathscr{O}\left(10^{15} \mathrm{TeV}\right)$. The 4D space time is extended by one extra dimension, which is hidden from the Standard Model (SM) particles and forces by confining them to a 4D subspace (SM brane). The extra dimension may be large, in contrast to [2]. Only gravity is allowed to propagate into the extra dimension. The hierarchy is generated by a specifically chosen geometry ("warped" geometry), which leads to the localisation of gravity close to a second brane (Planck brane) at a distance $r_{0}$ away from the SM brane. Due to the small overlap with the SM brane, gravity appears weak. Local fluctuations of the inter brane distance manifest in a new particle, the radion. The radion acquires a mass due to the stabilisation of the brane distance [3]. To avoid new hierarchies, the mass is expected to be $\mathscr{O}(\mathrm{TeV})$. The radion is a scalar particle. Since the radion carries the quantum numbers of the Higgs boson, the radion and the Higgs boson may mix [4]. The mixing is controlled by the parameter $\xi$ (physical particles exist only for a limited range of $\xi$ ). Further parameters of the model are: the energy scale on the SM brane, $\Lambda_{\mathrm{W}}$, and the masses, $m_{\mathrm{r}}$ and $m_{\mathrm{h}}$, of the two mass eigenstates, the radion-like state, $r$, and the Higgs-like state, $\mathrm{h}$.

The radion couples to the trace of the energy momentum (EM) tensor. Thus, to first order the radion couples to massive particles with couplings proportional to the particle mass, and the Lorentz structure of the couplings is identical to that of the Higgs boson. However, the coupling strength of the radion is generally reduced by $v / \sqrt{6} \Lambda_{\mathrm{W}}$ w.r.t. the couplings of the SM Higgs boson, where $v$ denotes the vacuum expectation value of the Higgs field. At LEP2, both are predominantly produced in the "Higgsstrahlung" process, $\mathrm{e}^{+} \mathrm{e}^{-} \rightarrow \mathrm{Zr}$ or $\mathrm{Zh}$. The production of the radion-like and the Higgs-like states are complementary as seen in Figure 1a. Unlike the Higgs boson, which only couples to gluons via a top loop, the radion couples directly to gluon pairs due to the anomaly of the trace of the EM tensor. As a consequence, the radion decays mostly into gluon pairs. The 
branching ratio of the Higgs-like state into heavy quarks and leptons may be reduced depending on the mixing parameter $\xi$ while the branching ratio into gluon pairs is enhanced, which can be seen in Figure 1b.

The limits on the cross-section of the Higgsstrahlung process obtained at OPAL from searches for the SM Higgs boson [5], flavour independent searches for hadronically decaying Higgs bosons [6] and decay-mode independent searches for Higgs bosons [7] are used to restrict the parameter space of the RS model. This is explained in Section 2. A detailed description is given in [8].

Constraints of the RS model parameters have also been derived from the electroweak-precision observables e.g. [9], the measurement of anomalous magnetic moment of the muon [10], and direct searches for Kaluza-Klein Excitations e.g. [11].

\section{Experimental Constraints on the Randall-Sundrum Model}

Since the signatures of the radion-like and the Higgs-like states are similar to the signatures of the SM Higgs boson or neutral Higgs bosons of more general models, searches for a neutral Higgs boson also constrain the parameter space of the Randall-Sundrum (RS) model. The search for the SM Higgs boson covers the region of the parameter space, where the coupling of one of the scalars to bottom quarks is large. The flavour independent search becomes important in regions in which the coupling of the lighter scalar to gluons is significant. The region below $12 \mathrm{GeV}$ is covered by the decay mode independent search.

These searches have not revealed any significant excess of data over the background from Standard Model processes, and limits on the cross-section of the Higgsstrahlung process times the branching ratio of the scalar particle decay have been derived at the $95 \%$ confidence level.

Points in the parameter space of the RS model are considered excluded if the predicted crosssection times branching ratio for either the radion-like or the Higgs-like state exceeds the limit obtained from one of the Higgs boson searches. At each scan point, the search is chosen which yields the most restrictive expected limit.

In Figure $2 \mathrm{a}$ and $\mathrm{b}$, the minimum allowed mass of the radion-like state at the $95 \% \mathrm{CL}$ is shown (expected and observed limits). At each scan point spanned by the mixing parameter $\xi$ and the scale parameter $\Lambda_{\mathrm{W}}$, the minimum allowed mass of the radion-like state is search, where the masses of the Higgs-like state is varied in the range from $1 \mathrm{MeV}$ to $1 \mathrm{TeV}$.

The cross-section of the radion-like state vanishes for $\xi$ very close to the negative bound of the inaccessible region (the resolution of the Figures 2 is not sufficient to resolve this behaviour). A limit on $m_{\mathrm{r}}$ independent of $\xi$ cannot be derived. The cross-section decreases rapidly with increasing $\Lambda_{\mathrm{W}}$, since the couplings of the radion to SM particles are proportional to the inverse of $\Lambda_{\mathrm{W}}$. The analyses lose their sensitivity for $\Lambda_{\mathrm{W}} \gtrsim 0.8 \mathrm{TeV}$.

The mass of the Higgs-like state can be limited for all values of the mixing parameter $\xi$ and all $m_{\mathrm{r}}$ in the range $1 \mathrm{MeV}$ to $1 \mathrm{TeV}$. The expected and observed limits on the mass are displayed as a function of the scale parameter, $\Lambda_{\mathrm{W}}$, in Figure 3. The parameter space contains several theoretically inaccessible regions. Regions are accessible only if the mass difference is large enough (where the minimum mass difference depends on $\xi$ and $\Lambda_{\mathrm{W}}$ ). This leads to the steps in Figure 3 . The difference between the expected and observed limits may be artificially enhanced, if e.g. the observed value is just below an inaccessible region and the expected value just above. For all $\xi, m_{\mathrm{r}}$ and $\Lambda_{\mathrm{W}}$, the 
Higgs mass has to be larger than $58 \mathrm{GeV}$ at the $95 \%$ confidence level, where a limit of $54 \mathrm{GeV}$ is expected.

\section{Summary}

Limits on the Higgsstrahlung cross-section obtained from data recorded with the OPAL detector have been used to restrict the parameter space of the Randall-Sundrum model. The data exclude masses for the Higgs-like state below $58 \mathrm{GeV}$ for all scales $\Lambda_{\mathrm{W}} \geq 246 \mathrm{GeV}$, independent of the mixing between the radion and the Higgs boson, and of the radion mass. The analyses are sensitive to the radion for scales $\Lambda_{\mathrm{W}} \lesssim 0.8 \mathrm{TeV}$. No universal limit, independent of $\Lambda_{\mathrm{W}}, \xi$ and $m_{\mathrm{h}}$, on the mass of the radion-like state can be extracted.

\section{References}

[1] L. Randall and R. Sundrum, Phys. Rev. Lett. 83 (1999) 3370.

L. Randall and R. Sundrum, Phys. Rev. Lett. 83 (1999) 4690.

[2] N. Arkani-Hamed, S. Dimopoulos, and G. R. Dvali, Phys. Lett. B429 (1998) 263.

[3] C. Csáki, M. Graesser, L. Randall, and J. Terning, Phys. Rev. D62 (2000) 045015.

W. D. Goldberger and M. B. Wise, Phys. Lett. B475 (2000) 275.

W. D. Goldberger and M. B. Wise, Phys. Rev. Lett. 83 (1999) 4922.

[4] G. F. Giudice, R. Rattazzi, and J. D. Wells, Nucl. Phys. B595 (2001) 250.

C. Csáki, M. L. Graesser, and G. D. Kribs, Phys. Rev. D63 (2001) 065002.

[5] OPAL Collaboration, G. Abbiendi et al., Eur. Phys. J. C26 (2003) 479.

[6] OPAL Collaboration, G. Abbiendi et al., Phys. Lett. B597 (2004) 11.

[7] OPAL Collaboration, G. Abbiendi et al., Eur. Phys. J. C27 (2003) 311.

[8] OPAL Collaboration, G. Abbiendi et al., Phys. Lett. B609 (2005) 20-34.

[9] J. F. Gunion, M. Toharia, and J. D. Wells, Phys. Lett. B585 (2004) 295.

[10] P. K. Das, hep-ph/0407041.

[11] H. Davoudiasl, J. L. Hewett, and T. G. Rizzo, Phys. Rev. D63 (2001) 075004. 

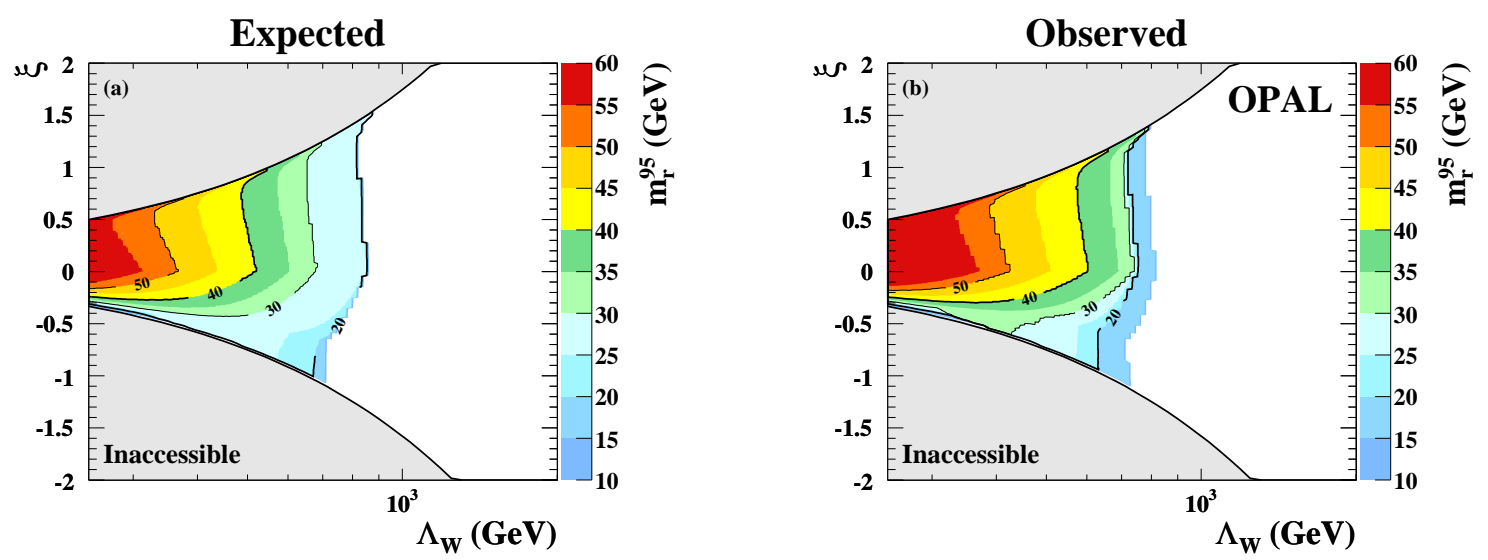

Figure 2: Expected (a) and observed (b) lower limits on the mass of the radion-like state, $m_{\mathrm{h}}$ and $m_{\mathrm{r}}$, as a function of the mixing parameter $\xi$ and the scale parameter $\Lambda_{\mathrm{W}}$. Inside each shaded region, the obtained lower mass limit is equal or larger than the value indicated by the code on the right. The regions in the upper and lower left corner are inaccessible by the theory.

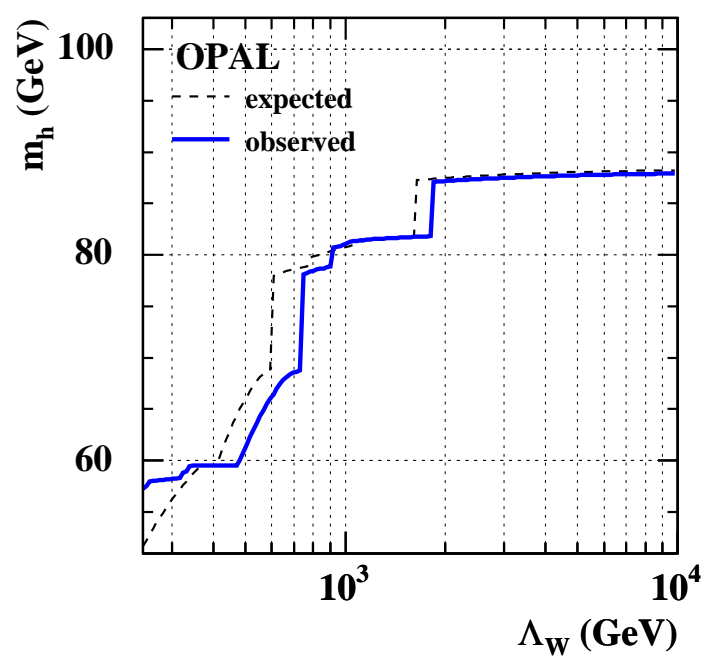

Figure 3: The lowest expected and observed limit on the Higgs boson mass as a function of the scale parameter $\Lambda_{\mathrm{W}}$ for all allowed $\xi$ and for masses of the radion-like state $m_{\mathrm{r}}$ in the range from $1 \mathrm{MeV}$ to $1 \mathrm{TeV}$. The analyses often lose their sensitivity close to the inaccessible region. If the region up to the inaccessible region is covered, the next allowed mass will be several $\mathrm{GeV}$ further away. This causes the step like structure. 